\title{
Fetal Abdominal Obesity Detected at 24 to 28 Weeks of Gestation Persists until Delivery Despite Management of Gestational Diabetes Mellitus (Diabetes Metab J 2021;45:547-57)
}

\author{
Wonjin Kim ${ }^{1,2, *}$, Soo Kyung Park ${ }^{3, *}$, Yoo Lee Kim ${ }^{1}$ \\ ${ }^{1}$ Division of Endocrinology and Metabolism, Department of Internal Medicine, CHA Gangnam Medical Center, CHA University School of Medicine, Seoul, \\ ${ }^{2}$ Yonsei University College of Medicine, Seoul, Korea, \\ ${ }^{3}$ Department of Biostatics and Data Science, University of Texas Health Science Center at Houston, Houston, TX, USA
}

We would like thank Professor Kyung-Soo Kim for his insightful comments on our article, "Fetal abdominal obesity detected at 24 to 28 weeks of gestation persists until delivery despite management of gestational diabetes mellitus," and the opportunity to discuss the issues raised.

First, fetal abdominal obesity (FAO) has been detected as early as 21 weeks of gestation in our unpublished results which was submitted elsewhere. Considering that FAO is mainly associated with fetal hyperinsulinemia and that an increase in fetal pancreatic insulin production is observed as early as 10 to 14 weeks of gestation in the mother with pre-gestational diabetes mellitus (GDM) [1], we assume that interventions such as lifestyle modifications related to diet and exercise should be initiated well before or early in pregnancy to reduce the risk of FAO. Further studies are necessary to clarify this.

Secondly, at the time of diagnosis of GDM, the risk of FAO in young and non-obese GDM was not higher than the risk in women with normal glucose tolerance (NGT), but the risk of FAO was significantly increased in those who were older and/ or obese with GDM [2]. Near term, although the risk of FAO in young and non-obese GDM was not higher than in NGT subjects, the risk of FAO in older and/or obese subjects with GDM was persistently higher than in NGT subject despite treatment [3]. We suggest that the risk of FAO in young and non-obese GDM remained as low as NGT with appropriate treatment, but that the increased risk for FAO in older and/or obese GDM was not improved. Fetal hyperinsulinemia was already established at diagnosis of GDM in high risk older and/ or obese women and was thought to persist until delivery, resulting in FAO near term.

Finally, this study has limitations mainly due to its retrospective nature. Because we focused on whether FAO in high-risk women with GDM improves or persists under the current best practice guidelines, there is not enough data on factors affecting fetal growth such as maternal lipid profile [4], as you mentioned. More extensive research is needed to explain the findings we observed.

In conclusion, FAO associated with fetal hyperinsulinemia begins much earlier during pregnancy than other issues related to large for gestational age or macrosomia. Therefore, interventions before or during early pregnancy are recommended to prevent FAO.

\section{CONFLICTS OF INTEREST}

No potential conflict of interest relevant to this article was reported.

\footnotetext{
Corresponding author: Yoo Lee Kim (D) https://orcid.org/0000-0002-9025-9774 Division of Endocrinology and Metabolism, Department of Internal Medicine, CHA Gangnam Medical Center, CHA University School of Medicine, 566 Nonhyeon-ro, Gangnam-gu, Seoul 06135, Korea E-mail: ylkim@cha.ac.kr

*Wonjin Kim and Soo Kyung Park contributed equally to this study as first authors.
}

This is an Open Access article distributed under the terms of the Creative Commons Attribution Non-Commercial License (https://creativecommons.org/licenses/by-nc/4.0/) which permits unrestricted non-commercial use, distribution, and reproduction in any medium, provided the original work is properly cited. 


\section{REFERENCES}

1. Poissonnet CM, Burdi AR, Bookstein FL. Growth and development of human adipose tissue during early gestation. Early Hum Dev 1983;8:1-11.

2. Kim W, Park SK, Kim YL. Gestational diabetes mellitus diagnosed at 24 to 28 weeks of gestation in older and obese women: is it too late? PLoS One 2019;14:e0225955.

3. Kim W, Park SK, Kim YL. Fetal abdominal obesity detected at
24 to 28 weeks of gestation persists until delivery despite management of gestational diabetes mellitus. Diabetes Metab J 2021;45:547-57.

4. Kim SY, Song YS, Kim SK, Cho YW, Kim KS. Postprandial free fatty acids at mid-pregnancy increase the risk of large-for-gestational-age newborns in women with gestational diabetes mellitus. Diabetes Metab J 2021 Aug 9 [Epub]. https://doi. org/10.4093/dmj.2021.0023. 